\title{
Preprocessing Techniques for High-Efficiency Data Compression in Wireless Multimedia Sensor Networks
}

\author{
Junho Park ${ }^{1}$ and Jaesoo Yoo ${ }^{2}$ \\ ${ }^{1}$ Agency for Defense Development, Daejeon 305-600, Republic of Korea \\ ${ }^{2}$ School of Information and Communication Engineering, Chungbuk National University, Cheongju, \\ Chungbuk 361-763, Republic of Korea \\ Correspondence should be addressed to Jaesoo Yoo; yjs@chungbuk.ac.kr
}

Received 29 August 2014; Accepted 3 November 2014

Academic Editor: Seungmin Rho

Copyright (C) 2015 J. Park and J. Yoo. This is an open access article distributed under the Creative Commons Attribution License, which permits unrestricted use, distribution, and reproduction in any medium, provided the original work is properly cited.

\begin{abstract}
We have proposed preprocessing techniques for high-efficiency data compression in wireless multimedia sensor networks. To do this, we analyzed the characteristics of multimedia data under the environment of wireless multimedia sensor networks. The proposed preprocessing techniques consider the characteristics of sensed multimedia data to perform the first stage preprocessing by deleting the low priority bits that do not affect the image quality. The second stage preprocessing is also performed for the undeleted high priority bits. By performing these two-stage preprocessing techniques, it is possible to reduce the multimedia data size in large. To show the superiority of our techniques, we simulated the existing multimedia data compression scheme with/without our preprocessing techniques. Our experimental results show that our proposed techniques increase compression ratio while reducing compression operations compared to the existing compression scheme without preprocessing techniques.
\end{abstract}

\section{Introduction}

In recent, with the development of hardware technologies and monitoring schemes, the applications for gathering multimedia data such as sound and image using multimedia sensors have been increased $[1,2]$. As the multimedia data are very large over simple data in traditional sensor networks, the network lifetime of the sensor network is significantly reduced due to excessive energy consumption in particular nodes for transmitting the multimedia data [3]. In addition, the multimedia data increase the data transmission time and decline the data reception ratio. Consequently, the existing schemes based on the traditional sensor networks are not suitable for the environments to collect the multimedia data $[3,4]$.

It is necessary to use compression schemes to alleviate such problems. However, most of the existing compression schemes for sensor data are based on signal compression such as wavelet and variable quantization and code compression [5]. These studies are not suitable for the environments based on wireless sensor networks. The compression schemes for wireless multimedia sensor networks are at an early stage. Considering this, a novel compression scheme based on the Chinese remainder theorem for the multimedia data has been proposed in [6]. The compression scheme based on the Chinese remainder theorem considered the characteristics and limitation of wireless multimedia sensor networks. However, the existing compression scheme still suffers from energy consumption in the wireless multimedia sensor network with the limited energy.

In this paper, we propose preprocessing techniques for high-efficiency data compression in wireless multimedia sensor networks. The proposed techniques consider the characteristics of sensed multimedia data to perform the first stage preprocessing by deleting the low priority bits that do not affect the image quality. The second stage preprocessing is also performed for the undeleted high priority bits. By performing these two-stage preprocessing techniques, it is possible to reduce the multimedia data size in large.

The remainder of this paper is organized as follows. Section 2 overviews and analyzes the existing compression schemes. In Section 3, we present our preprocessing 
techniques for high-efficiency data compression in wireless multimedia sensor networks. Section 4 shows the simulated experiments and compares the existing scheme with the proposed scheme. Finally, we present concluding remarks in Section 5.

\section{Related Works}

The existing compression schemes for multimedia data can be categorized under 1st and 2nd generation schemes [7]. The 1st generation schemes such as DCT based compression [8], EZW [9], SPIHT [10], and EBCOT [11] emphasize more on how well the information contained in a transformed multimedia data is efficiently encoded whereas the 2 nd generation schemes such as Pyramidal [12], Directional Decomposition [13], Segmentation [14], and Vector Quantization [15] place more importance on how we can exploit and extract useful information from the multimedia data. However, the existing compression schemes require the high-computational power and large memory to process the compression. Because of the constrained hardware of a wireless sensor node, these studies are not suitable for the environments based on wireless sensor networks. Considering this, a compression scheme based on the Chinese remainder theorem for the multimedia data was proposed [6]. However, the existing compression scheme suffers from energy consumption in the wireless multimedia sensor network with the limited energy. Therefore, it is necessary to study a preprocessing technique considering the characteristics of the wireless multimedia sensor networks to improve the efficiency of compression scheme.

\section{The Preprocessing Techniques for High-Efficiency Data Compression}

In this paper, for the maximization of compression efficiency, we propose the preprocessing techniques that consist of dynamic area extraction and bit plane deletion before conducting compression scheme. By doing so, the proposed scheme reduces data compression operation, improves compression ratio, and minimizes energy consumption generated from data transmission by transmitting the remaining data instead of original multimedia sensor data.

3.1. Preprocessing Technique (1): Extracting the Dynamic Area. In general, the wireless multimedia sensor networks require the installation costs and distribute the few multimedia sensor nodes and many general sensor nodes together. As shown in Figure 1, the first detection is done through general scalar sensor nodes. When an abnormal signal (event) is detected, the near multimedia sensor nodes perform the second detection for the detailed monitoring. The position of a sensor node distributed to the network is fixed. As the multimedia sensor node is designed for the unidirectional shooting, it continuously shoots the image data of the space so that the abnormal signal is detected by adjusting the shooting angle and transmits the sensed data to the base station.

Figure 2 shows the characteristics of sensed images in the wireless multimedia sensor networks. As shown in

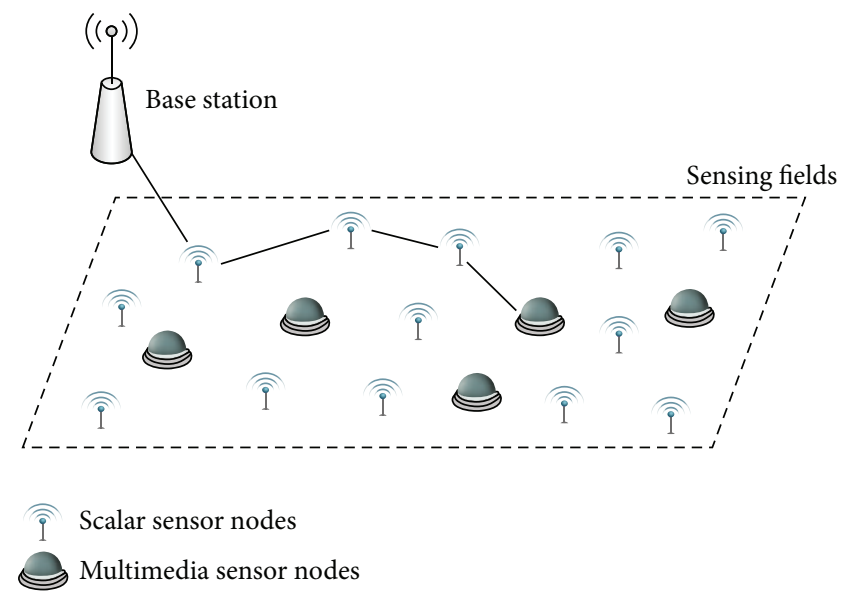

FIGURE 1: Deployment of wireless multimedia sensor networks.

the Figure 2, the wireless multimedia sensor node continuously shoots the image of space that the abnormal signal is detected so that a dynamic area and a static area with a considerable size occur according to the time. By considering the characteristics, the transmission of the whole sensed data causes the unnecessary communication cost. It also reduces network lifetime. In order to solve such problems, the proposed scheme extracts and compresses the high efficient dynamic area.

First of all, it is important to detect the dynamic area in the sensed data. As it needs a high arithmetic operation cost to compare all pixels in the detection stage of the dynamic area, it is not efficient. Therefore, the proposed scheme generates the virtual comparison block through the pixel clustering and the detection of the image change and uses it as the transmission unit as shown in Figure 3. As the virtual comparison block is utilized as a transmission unit, there should be no effect on the compression based on the Chinese remainder theorem [3]. The conditions of the virtual comparison block are as follows by the definition of compression scheme based on the Chinese remainder theorem.

Condition 1. As the continuous decimal numbers to utilize the Chinese remainder theorem are at least two or more, it must be possible to express the comparison block data with two continuous decimal numbers.

Condition 2. As the actual transmission data to the base station is the remainder, the remainder is below the maximum expression range of a specific variable type for the maximum efficiency.

Condition 3. For the easy restoration of original data and block data, it is a structure of square which is applicable for the QCIF format.

By the conditions above, the maximum block size for possible utilization is $3 \times 3$ pixels (the proof is omitted due to the space limit). 


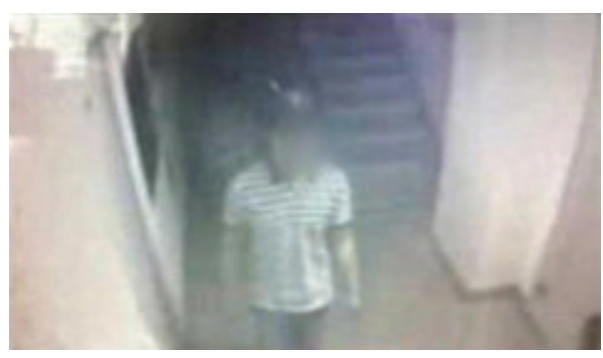

(a) $t_{0}$

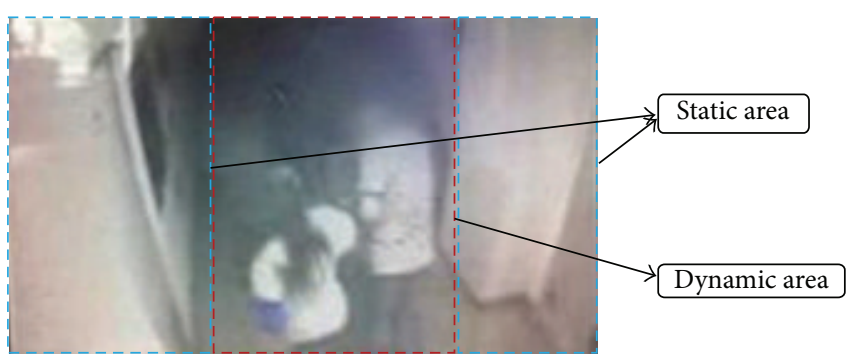

(b) $t_{1}$

FIGURE 2: Characteristic of sensed images in the wireless multimedia sensor networks.

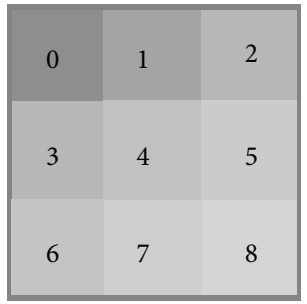

(a) Virtual comparison block \#n

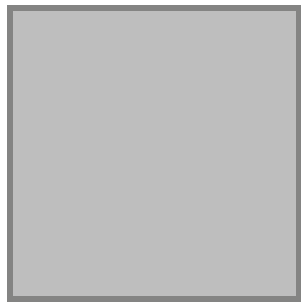

(b) Average value of virtual comparison block \#n

FIgURE 3: Virtual comparison block and clustering operation.

The virtual comparison block with a grid shape is generated to the sensed image. As in (1), the proposed scheme clusters the colors of all pixels inside the corresponding virtual comparison block and computes their average values. At this time, if the average values of the colors in the virtual comparison blocks of the initially sensed images and the currently sensed images are above the threshold value, they should be recognized as the dynamic areas as follows:

$$
\begin{aligned}
& \text { |firstImgBlock \#n.avrValue - curImgBlock \#n.avrValue } \\
& \quad>\alpha \text {. }
\end{aligned}
$$

Figure 4 shows the detection of the dynamic area and the extraction of the transmission area. Through the comparison of average values of colors in the virtual comparison block, the dynamic area that must be transmitted is recognized. The corresponding dynamic area is compressed based on the Chinese remainder theorem to transmit it to the base station. At this time, when the packets are transmitted in the virtual comparison block unit, unnecessary data transmission occurs due to header and trailer. To solve such a problem, the proposed scheme transmits the dynamic area in a single packet through the establishment and extraction of the maximum transmission area. Through this process, the proposed scheme minimizes the energy consumption by the partial compression and transmission of the dynamic area.

\subsection{Preprocessing Technique (2): Deleting Bit Plane Data.} The sensor node is driven based on the limited energy and computing performance so that it needs an additional compression module to perform the data compression. However, it requires a lot of installation costs. Considering this, transmitting the original data was common in the existing scheme. Figure 5 shows the structure of multimedia sensor data in wireless multimedia sensor networks. The image data sensed from the wireless multimedia sensor network has a data bit structure of the pixel unit, and each pixel has eight bits (0 255) per color channel (R, G, B) as shown in Figure 5. The same bits are bound together to compose a bit plane. As a result, the color image is composed of 24 bit planes in total. While the data sizes of the bit plane are equivalent, the amount of their expressible information and the importance are different. For example, each bit of the most significant 7 bit plane can express 128, but each bit of the lowest significant 0 bit plane can express only 1 . In other words, the most significant bit plane shows a big color change and the lowest bit plane shows the small color change.

Based on this analysis, Figure 6 shows the changes in image quality according to the bit plane deletion. When the most significant bit plane is deleted, serious damages to the quality of the original data occur. However, when the low significant bit plane data is deleted, it does not largely affect the quality of the original data. From the result, when all bit plane data cannot be transmitted, the high quality image can be maintained by deleting data near the lowest bit plane. In the proposed scheme, the preprocessing (2) is performed for the image data based on this fact.

According to the result from the preprocessing (2), few bit plane data are selected as transmission data. As a result, the proposed scheme minimizes the energy consumption and increases the lifetime of a whole network by utilizing this kind of preprocessing techniques.

\section{Performance Evaluation}

We have developed a simulator based on JAVA to evaluate the existing multimedia data compression scheme [6] with/without our preprocessing techniques. This simulation was carried out by constructing the performance evaluation environment shown in Table 1.

In order to carry out performance evaluation, we utilized the uncompressed MPEG-4 standard image provided by Xiph.org [16]. Figure 7 shows the sample images of uncompressed MPEG- 4 standard images. This simulation was done 


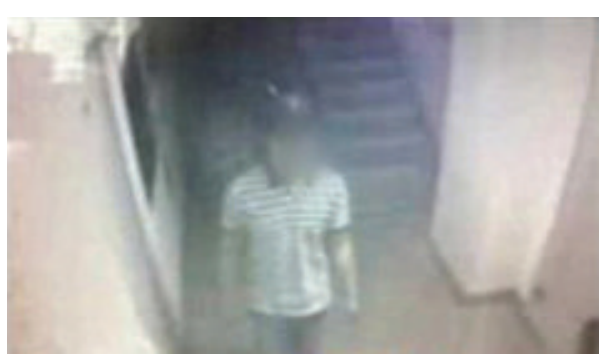

(a) $t_{0}$

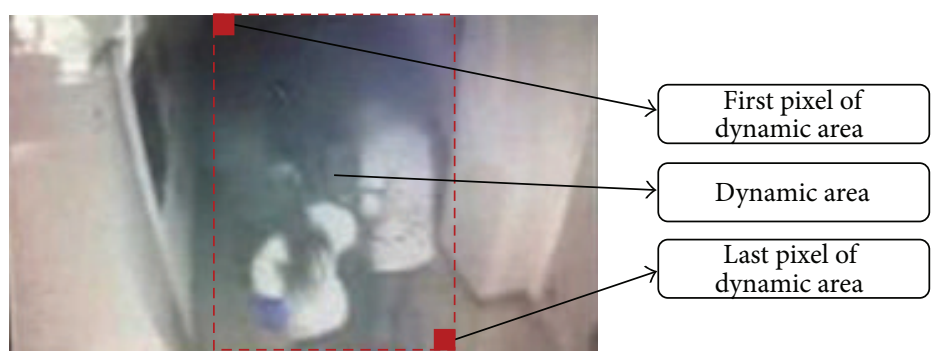

(b) $t_{1}$

FIgURE 4: Detection of the dynamic area and extraction of the transmission area.

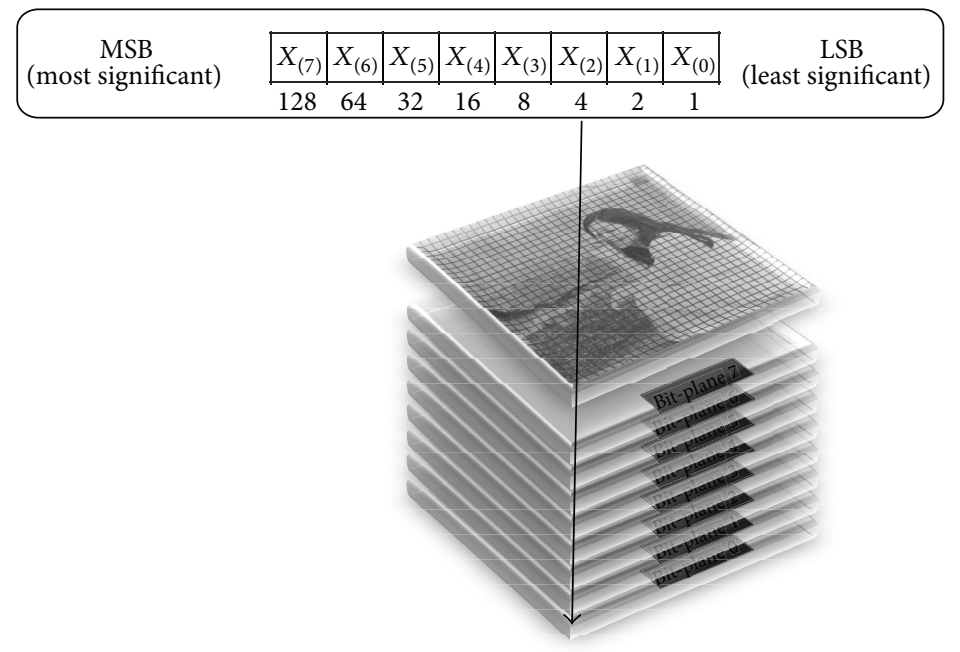

FIGURE 5: Structure of multimedia sensor data in wireless multimedia sensor networks.

TABLE 1: Simulation parameters.

\begin{tabular}{lc}
\hline Parameters & Values \\
\hline Multimedia data & $\begin{array}{c}\text { Uncompressed MPEG-4 } \\
\text { standard images provided by } \\
\text { Xiph.org and CCTV image }\end{array}$ \\
$\begin{array}{l}\text { Length of multimedia data } \\
\text { (frames })\end{array}$ & 50 \\
$\begin{array}{l}\text { Format of multimedia data } \\
(\mathrm{px} \times \mathrm{px})\end{array}$ & $\mathrm{CIF}(352 \times 288)$ \\
\hline
\end{tabular}

targeting the image having CIF (full CIF) and QCIF (quarter CIF) resolution which are generally gathered by wireless multimedia sensor networks. In Table 2, the image classification indicates spatial complexity and motion amount, in which " $\mathrm{A}$ " is the image expressing a tiny level of spatial complexity and movement while " $B$ " signifies the image expressing a midlevel of spatial complexity and movement [17]. In addition, the wireless multimedia sensor networks have a distribution characteristic and monitoring characteristic similar to that of closed circuit TV (CCTV). Therefore, in this simulation, the performance evaluation was conducted by collecting images of actual CCTV. The relevant images are CCTV images [18] of the fire occurrence at Busan Metro Daeti Station in Korea on
TABLE 2: Characteristics of uncompressed MPEG-4 standard images [16].

\begin{tabular}{lcccc}
\hline & $\begin{array}{c}\text { Resolution } \\
(\mathrm{px} \times \mathrm{px})\end{array}$ & $\begin{array}{c}\text { Size of images } \\
(\text { Mbytes })\end{array}$ & Frames & Class \\
\hline (a) Akiyo & CIF & CIF (44) & 300 & A \\
& $\begin{array}{l}(352 \times 288) \\
\text { QCIF } \\
(176 \times 144)\end{array}$ & QCIF (11) & & \\
\hline & CIF & CIF (44) & 300 & B \\
(b) News & $(352 \times 288)$ & & \\
& QCIF & QCIF (11) & & \\
\hline
\end{tabular}

August 27, 2012, and for simulation we converted the format to CIF and QCIF. Figure 8 shows the sample images of CCTV images.

Figure 9 shows the compression ratio per frame to standard image and CCTV image of the proposed scheme with preprocessing techniques. Performance evaluation was carried out by grafting preprocessing technique (1) and preprocessing technique (2) onto the existing scheme. Preprocessing technique (1) indicates dynamic area extraction and preprocessing technique (2) \#2, \#4, and \#6 indicate 


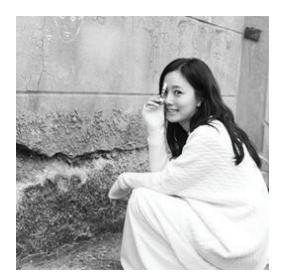

11111111

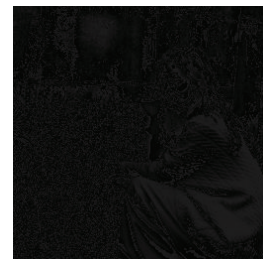

00011111

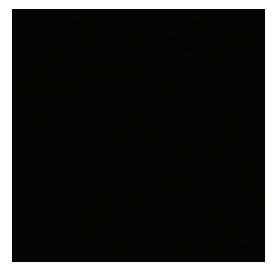

00000011

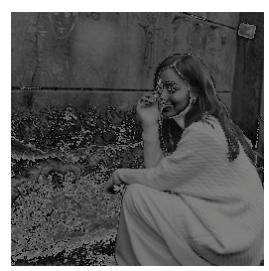

01111111

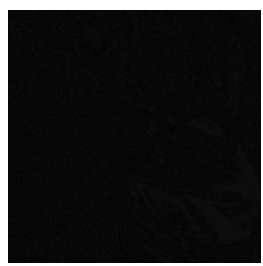

00001111

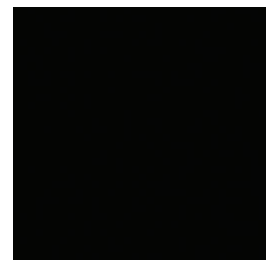

00000001

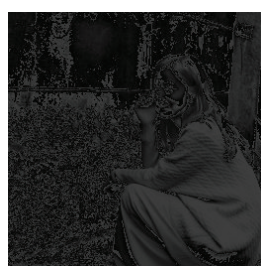

00111111

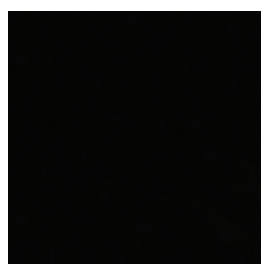

00000111

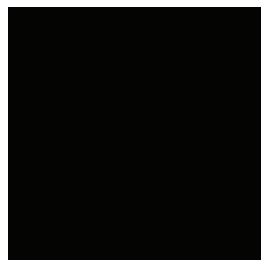

00000000

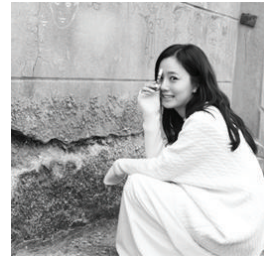

11111111

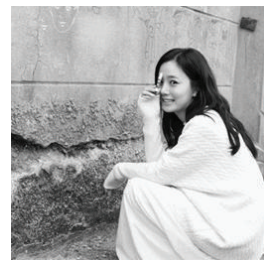

11111000

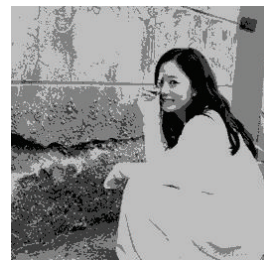

11000000

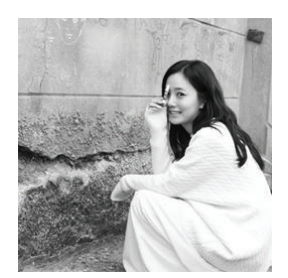

11111110

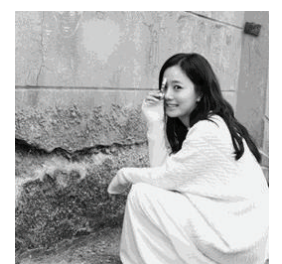

11110000

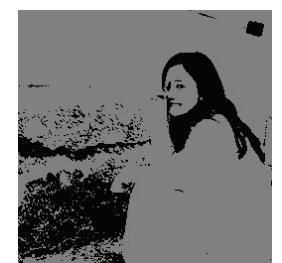

10000000

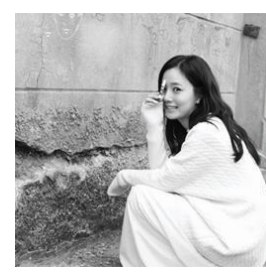

11111100

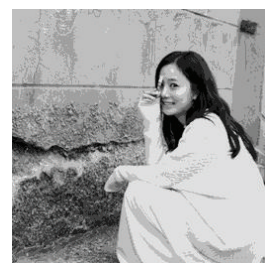

11100000

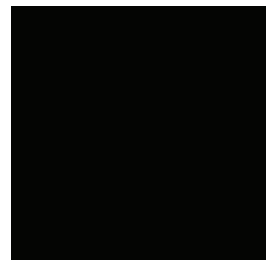

00000000

(a) Deletion of the most significant bit plane data

FIGURE 6: Changes in image quality according to the bit plane deletion.

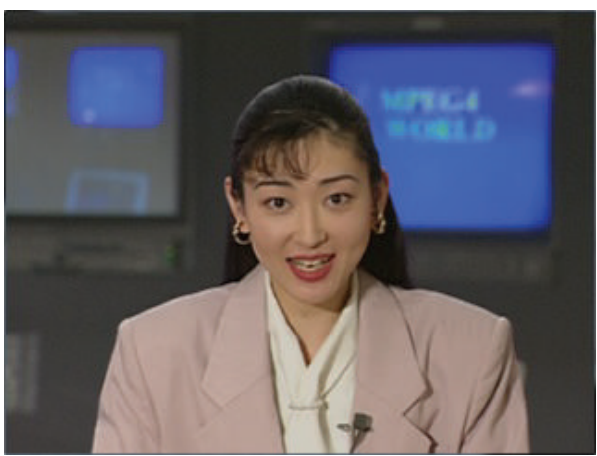

(a) Akiyo

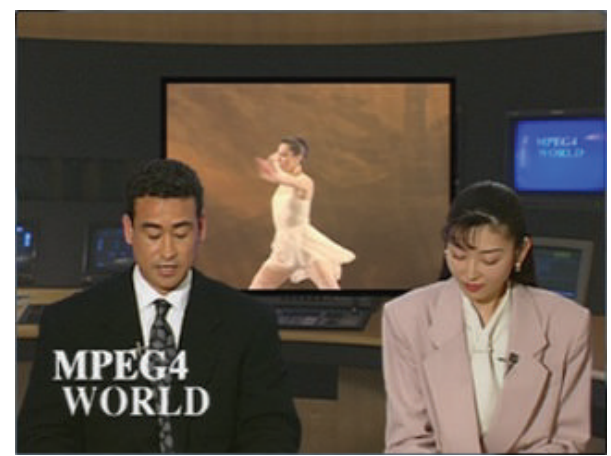

(b) News

FIGURE 7: Sample images of uncompressed MPEG-4 standard images.

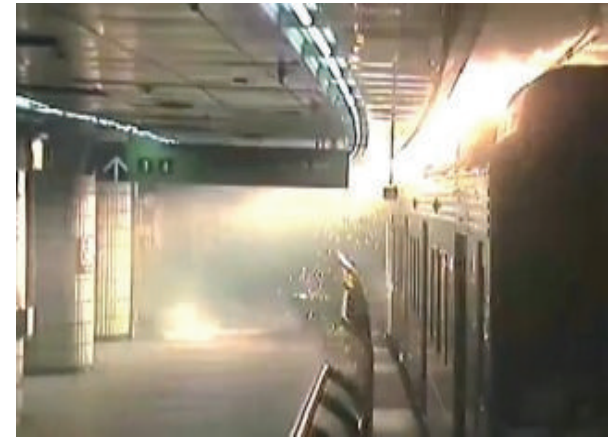

FIgURE 8: Sample images of CCTV image. the number deleting the bit plane, respectively. According to the experimental result, in case preprocessing technique (1) is applied, the compression ratio improves as the area for compression is reduced by extracting dynamic area while transmitting the relevant area by compressing the area only. In addition, in case preprocessing technique (2) is applied, the compression ratio also improves since the number of bit planes for compression is reduced by adjusting the quality of multimedia sensor data through the deletion of bit planes according to applications. In case 1 bit plane is deleted, preprocessing technique (2) improves compression efficiency by $12.5 \%$ on the basis of grayscale image but, as much, 


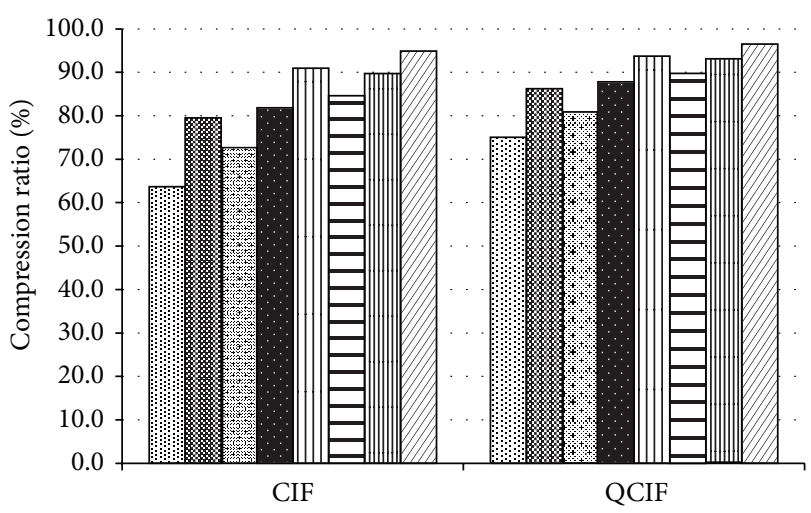

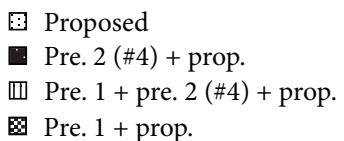

凹 Pre. $2(\# 6)+$ prop.

$\square$ Pre. $1+$ pre. $2(\# 6)+$ prop.

国 Pre. $2(\# 2)+$ prop.

日 Pre. $1+$ pre. $2(\# 2)+$ prop.

(a) Sample images

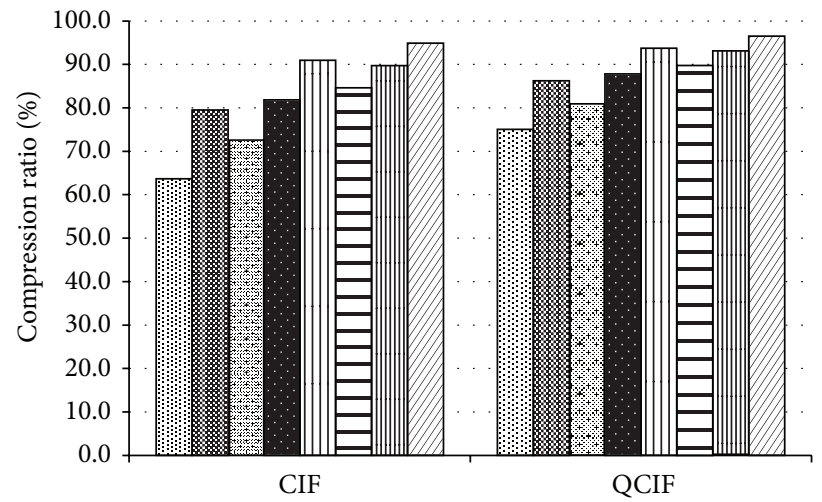

1 Proposed
Pre. $2(\# 4)+$ prop.
四 Pre. $1+$ pre. $2(\# 4)+$ prop.
2 Pre. $1+$ prop.

$\square$ Pre. $2(\# 6)+$ prop.

$\checkmark$ Pre. $1+$ pre. $2(\# 6)+$ prop.

图 Pre. $2(\# 2)+$ prop.

$\boxminus$ Pre. $1+$ pre. $2(\# 2)+$ prop.

(b) CCTV image

FIGURE 9: Compression ratio per frame with preprocessing techniques.

image information is lost; therefore, proper setup should be done according to application. In addition, in the event of grafting preprocessing technique (1) onto CCTV image, image change severely comes up, which causes dynamic area to be wide extracted relatively, and consequently it does not serve to improve compression efficiency to a larger extent than optimized image. Nonetheless, preprocessing technique (2) belongs to arithmetic operation deleting bit plane, causing no big impact. As a result, the existing scheme with preprocessing technique (1) and the existing scheme with preprocessing technique (2) improve the data compression ratio by about $25 \%$ and $42.9 \%$, respectively, over the existing scheme without preprocessing techniques. In addition, when preprocessing technique (1) and preprocessing technique (2) were simultaneously grafted, the existing scheme improves the data compression ratio by about $49.2 \%$ over the existing scheme without preprocessing techniques.

Figure 10 shows the compression arithmetic operation quantity in the existing scheme with preprocessing techniques. Preprocessing technique (1) requires per-pixel comparison operation with the previous image in order to extract the dynamic area, making it inevitable to carry out largescale arithmetic operation. In order to reduce this, perblock data comparison is performed, but it also requires large-scale arithmetic operations. In the event of grafting preprocessing technique (2), the compression operation quantity is decreased, which can be explained by the fact that compression operations decrease as much because the bit plane deleted by virtue of simple deletion operation does not require compression operations. Preprocessing technique (2) gets improved in compression efficiency by $12.5 \%$ on the basis of grayscale image when 1 bit plane is deleted. However, since the loss of image information can occur, it is important to properly set up according to applications.

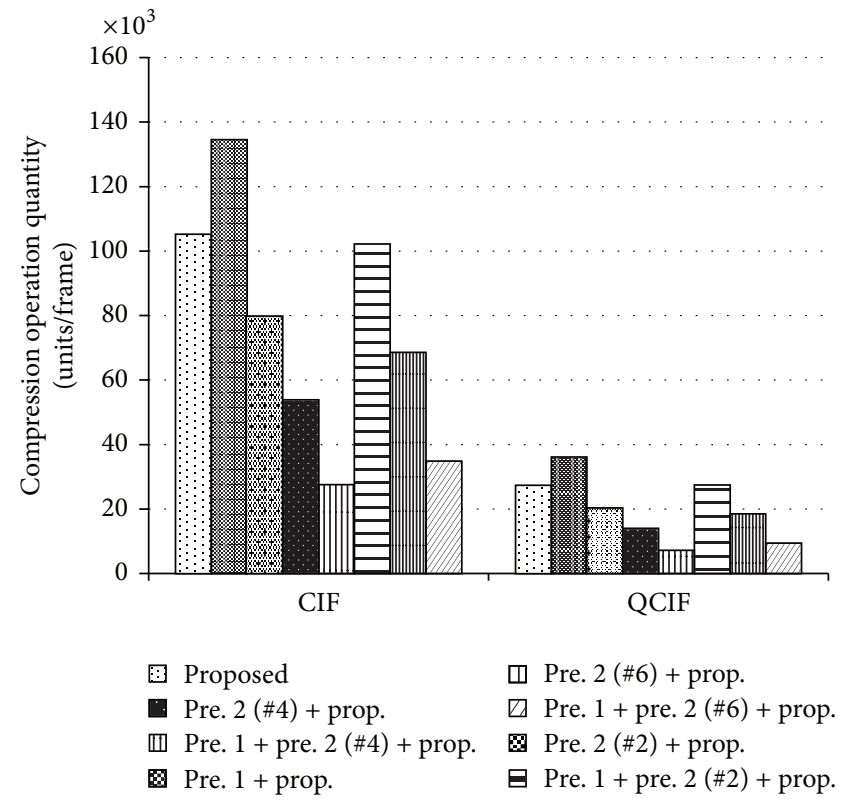

FIGURE 10: Compression operation quantity with preprocessing techniques.

\section{Conclusion}

In this paper, we have proposed preprocessing techniques for high-efficiency data compression in wireless multimedia sensor networks. To do this, we analyzed the characteristics of multimedia data under the environment of wireless multimedia sensor networks. The proposed scheme considers the characteristics of sensed multimedia data to perform the first compression by deleting the low priority bits that do not affect the image quality. The second stage compression is also 
performed based on the Chinese remainder theorem for the undeleted high priority bits. By performing this two-stage compression, it is possible to reduce the multimedia data size in large. To show the superiority of our techniques, we simulated the existing multimedia data compression scheme with/without our preprocessing techniques. As a result, it was shown through performance evaluation that the proposed preprocessing scheme significantly increased compression ratio while reducing compression operation comparing to the existing compression schemes without preprocessing techniques. In the future work, we plan to study the effective transmission scheme considering the characteristics of compression data.

\section{Conflict of Interests}

The authors declare that there is no conflict of interests regarding the publication of this paper.

\section{Acknowledgments}

This research was supported by the MSIP (Ministry of Science, ICT and Future Planning), Korea, under the ITRC (Information Technology Research Center) Support Program (NIPA-2014-H0301-14-1022) supervised by the NIPA (National IT Industry Promotion Agency) and the C-ITRC (Convergence Information Technology Research Center) Support Program (NIPA-2014-H0401-14-1007) supervised by the NIPA (National IT Industry Promotion Agency), by the ICT R \& D Program of MSIP/IITP [14-824-09-001], Development of High Performance Visual Big Data Discovery Platform for Large-Scale Real Time Data Analysis, and by the Ministry of Education (MOE) and National Research Foundation of Korea (NRF) through the Human Resource Training Project for Regional Innovation (no. 2013H1B8A2032298).

\section{References}

[1] S. M. Aziz and D. M. Pham, "Energy efficient image transmission in wireless multimedia sensor networks," IEEE Communications Letters, vol. 17, no. 6, pp. 1084-1087, 2013.

[2] P. Wang, R. Dai, and I. F. Akyildiz, "A differential codingbased scheduling framework for wireless multimedia sensor networks," IEEE Transactions on Multimedia, vol. 15, no. 3, pp. 684-697, 2013.

[3] I. F. Akyildiz, T. Melodia, and K. R. Chowdhury, "A survey on wireless multimedia sensor networks," Computer Networks, vol. 51, no. 4, pp. 921-960, 2007.

[4] T. Ma, M. Hempel, D. Peng, and H. Sharif, "A survey of energyefficient compression and communication techniques for multimedia in resource constrained systems," IEEE Communications Surveys and Tutorials, vol. 15, no. 3, pp. 963-972, 2013.

[5] W. C. Li, L. M. Ang, and P. S. Kah, "Survey of image compression algorithms in wireless sensor networks," in Proceedings of the International Symposium on Information Technology (ITSim '08), pp. 1-9, August 2008.

[6] H. Lee, J. Park, D. Seong, and J. Yoo, "An energy-efficient data compression and transmission scheme in wireless multimedia sensor networks," Journal of KIISE: Information Networking, vol. 39, no. 3, pp. 258-266, 2012.

[7] L. W. Chew, L.-M. Ang, and K. P. Seng, "Survey of image compression algorithms in wireless sensor networks," in Proceedings of the International Symposium on Information Technology (ITSim '08), pp. 1-9, IEEE, Kuala Lumpur, Malaysia, August 2008.

[8] D. Cruz, T. Ebrahimi, J. Askelof, M. Larsson, and C. Christopoulos, "Coding of still picture," in Applications of Digital Image Processing, vol. 4115 of Proceedings of SPIE, 2000.

[9] J. M. Shapiro, "Embedded image coding using zerotrees of wavelet coefficients," IEEE Transactions on Signal Processing, vol. 41, no. 12, pp. 3445-3462, 1993.

[10] A. Said and W. A. Pearlman, "A new, fast, and efficient image codec based on set partitioning in hierarchical trees," IEEE Transactions on Circuits and Systems for Video Technology, vol. 6, no. 3, pp. 243-250, 1996.

[11] D. Taubman, "High performance scalable image compression with EBCOT," IEEE Transactions on Image Processing, vol. 9, no. 7, pp. 1158-1170, 2000.

[12] P. J. Burt and E. H. Adelson, "The Laplacian pyramid as a compact image code," in Proceedings of the Korean Institute of Information Scientists and Engineers, vol. 31, pp. 532-540, 1983.

[13] A. Ikonomopoulos and M. Kunt, "High compression image coding via directional filtering," Signal Processing, vol. 8, no. 2, pp. 179-203, 1985.

[14] L. Ghouti, A. Bouridane, and M. K. Ibrahim, "Image compression using texture modeling," in Proceedings of the IEEE International Symposium on Circuits and Systems (ISCAS '05), vol. 3, pp. 2313-2316, May 2005.

[15] G. F. McLean, "Vector quantization for texture classification," IEEE Transactions on Systems, Man and Cybernetics, vol. 23, no. 3, pp. 637-649, 1993.

[16] Xiph.Org Foundation, 2013, http://www.xiph.org/.

[17] Xiph.org Video Test Media, 2013, http://media.xiph.org/video/ derf/.

[18] Fire Occurrence at Busan Metro Daeti Station in Korea on August 27, 2012, 2012, http://www.youtube.com/watch?v=Q-4KbAFEiEI. 

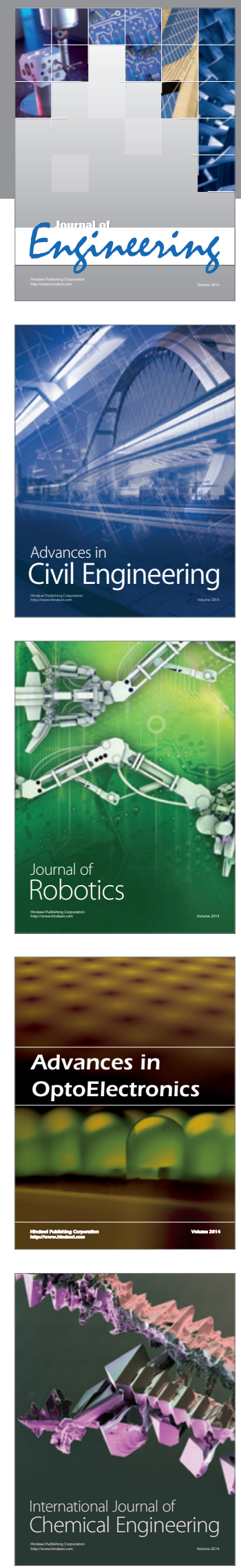

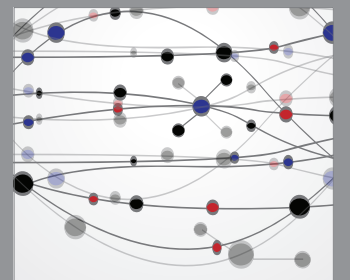

The Scientific World Journal
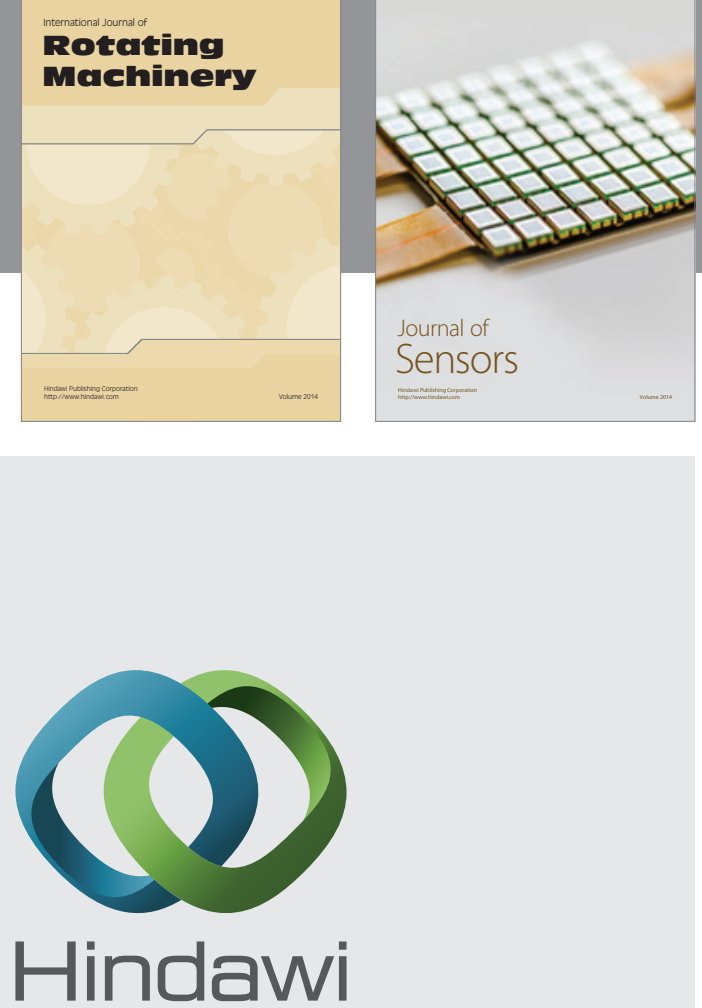

Submit your manuscripts at http://www.hindawi.com
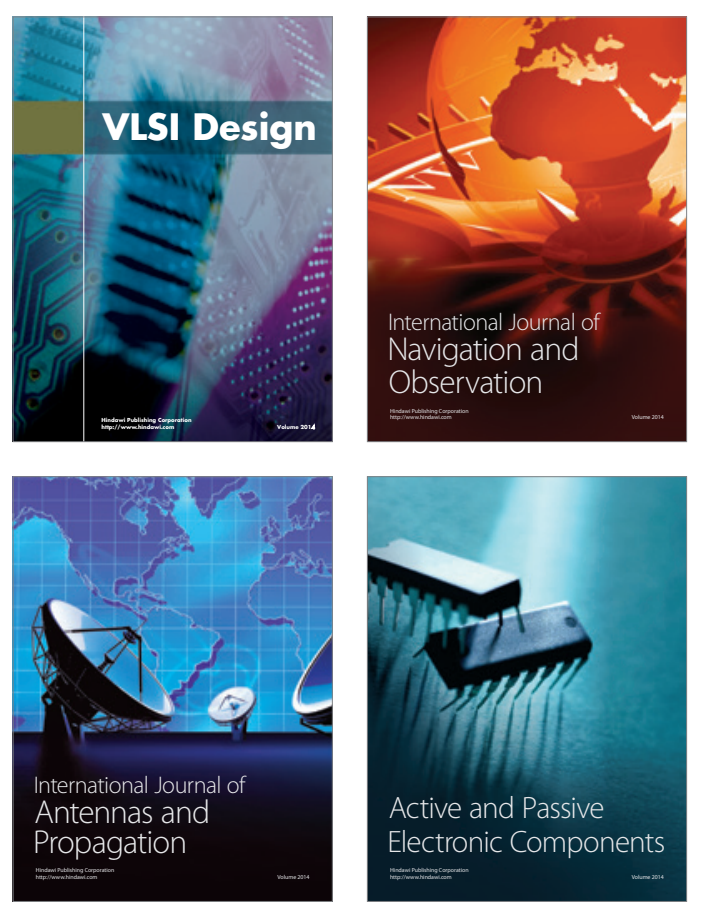
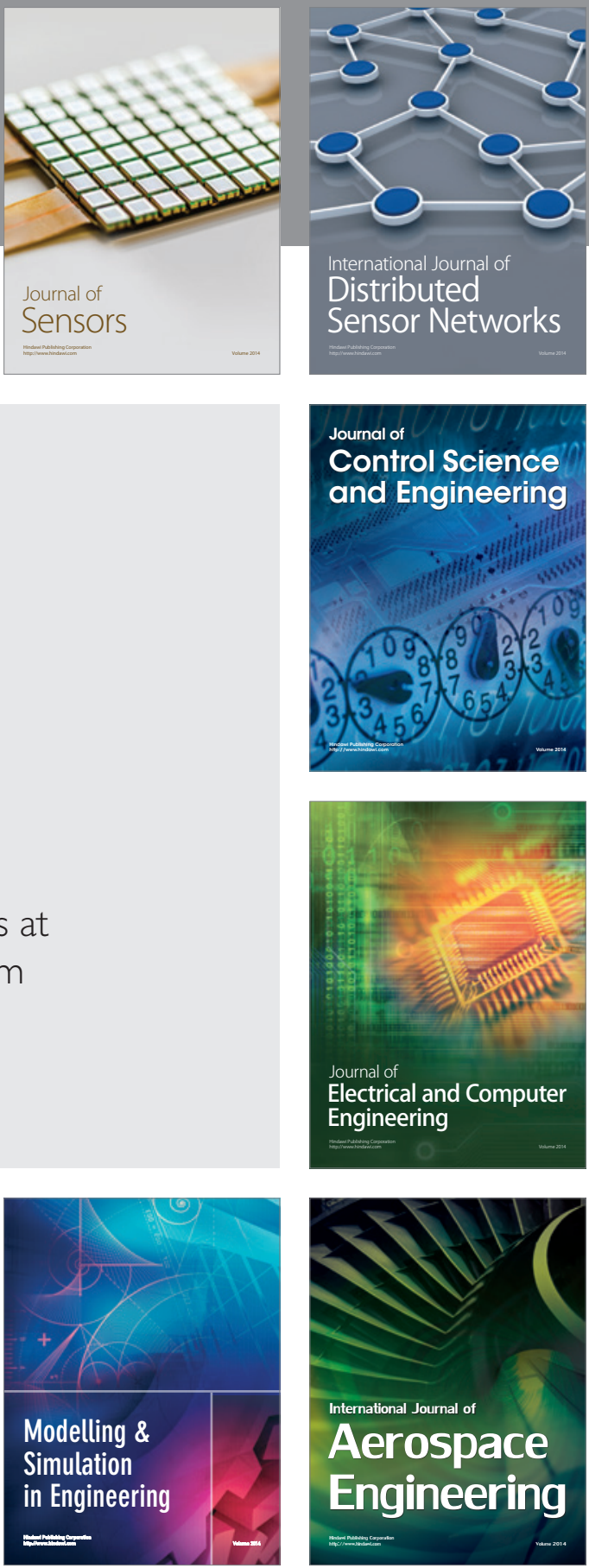

Journal of

Control Science

and Engineering
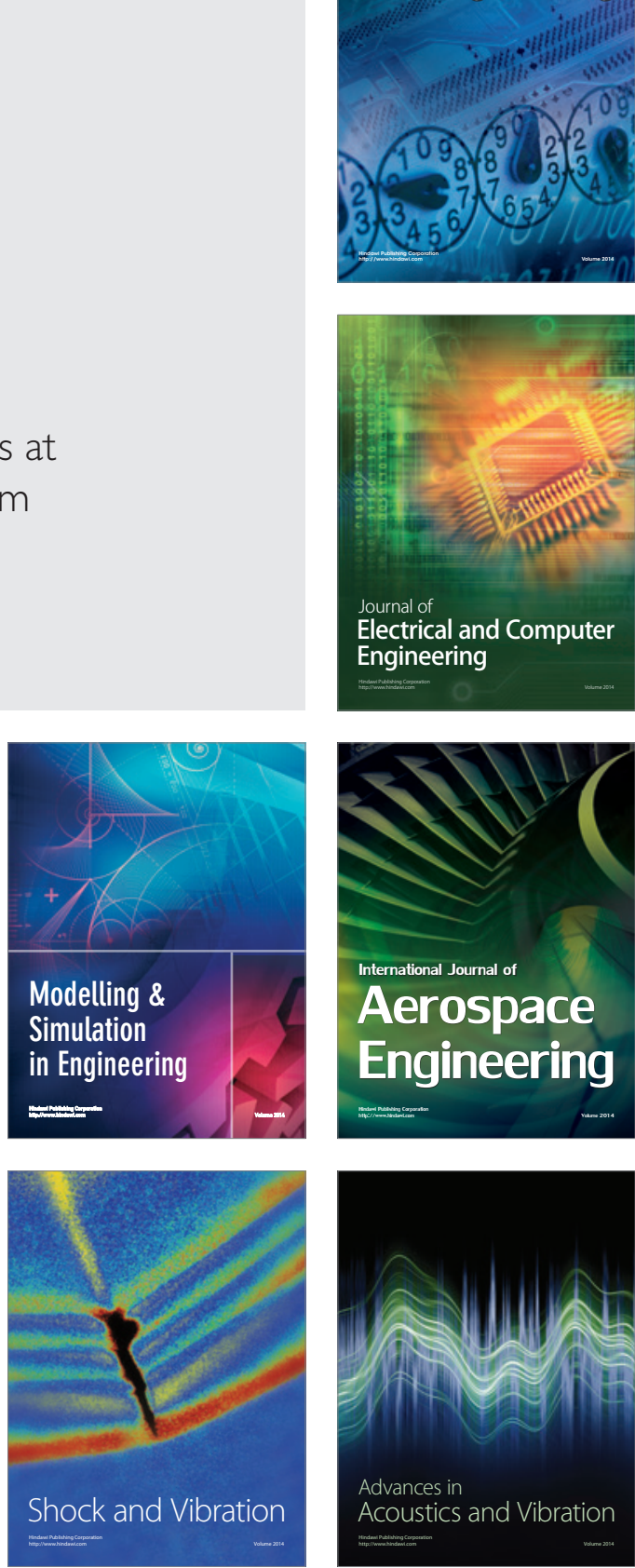